East African Medical Journal Vol. 82 No. 3 March 2005

PREVALENCE AND RISK FACTORS FOR VAGINAL CANDIDIASIS AMONG WOMEN SEEKING PRIMARY CARE FOR GENITAL INFECTIONS IN DAR ES SALAAM, TANZANIA

L. A. Namkinga, BPharm, MSc, PhD, Applied Microbiology Unit, University of Dar es Salaam, Tanzania, M.I.N. Matee, MD, MSc, PhD, Department of Microbiology and Immunology and A. K. Kivaisi, BSC, MSc, PhD, Applied Microbiology Unit, University of Dar es Salaam, Tanzania and C. Moshiro, BSc, MSc, Department of Biostatistics, Institute of Public Health, Muhimbili University College of Health Sciences, P.O. Box 65347, Dar es Salaam, Tanzania

Request for reprints to: Prof. M.I.N. Matee, Department of Microbiology and Immunology, Muhimbili University College of Health Sciences, Faculty of Medicine, P.O. Box 65347, Dar es Salaam, Tanzania

\title{
PREVALENCE AND RISK FACTORS FOR VAGINAL CANDIDIASIS AMONG WOMEN SEEKING PRIMARY CARE FOR GENITAL INFECTIONS IN DAR ES SALAAM, TANZANIA
}

\author{
L.A. NAMKINGA, M.I.N. MATEE, A.K. KIVAISI and C. MOSHIRO
}

\begin{abstract}
Objective: To determine the prevalence and risk factors for vaginal candidiasis (VC) among women seeking primary care for genital infections.

Design: Cross-sectional study.

Setting: Ilala Municipal Hospital in Dar es Salaam, Tanzania.

Subjects: Four hundred and sixty four women presenting with complaints of genital infections. Results: Of the 464 women examined, $177(38.1 \%)$ had abnormal vaginal discharge, $68(14.7 \%)$ had genital ulcers, $272(58.6 \%)$ had genital pruritis, $18(3.9 \%)$ had genital warts and $58(12.5 \%)$ had chancre. The prevalencies of $\mathrm{VC}$, bacterial vaginosis, HIV, $T$ vaginalis, $N$. gonorrhoeae and syphilis were $45 \%, 48.4 \%, 22 \%, 93 \%, 1.5 \%$ and $4.3 \%$, respectively. The occurrence of $\mathrm{VC}$ was positively associated with $\mathrm{HIV},(\mathrm{OR}=1.81,95 \% \mathrm{CI}(1.0-2.67)$, bacterial vaginosis; $(\mathrm{OR}=\mathbf{2 . 6}, 95 \% \mathrm{CI}(\mathbf{1 . 7 - 3 . 9})$, genital pruritis; $(\mathrm{OR}=1.8 \mathrm{1}, 95 \% \mathrm{CI}(1.2-$ 2.7) genital discharge; $(\mathrm{OR}=1.867,95 \%(1.28-2.73)$ and negatively with T. vaginalis $(\mathrm{OR}=0.27,95 \% \mathrm{CI}(0.12-0.6)$, occupation $(\mathrm{OR}=0.65,95 \% \mathrm{CI}(0.35-0.86))$ and with education $(\mathrm{OR}=0.43,95 \% \mathrm{CI}(\mathbf{0 . 1 1 - 0 . 7 3 )})$. There were increased but non-significant odds for $\mathrm{VC}$ in patients with syphilis $(\mathrm{OR}=1.695 \% \mathrm{CI}(\mathbf{0 . 6}-4.3)$ and venereal warts $(\mathrm{OR}=2.5$ 95\% CI (0.92-6.8) VC was not associated with $N$. gonorrhoeae, genital ulcers, age at first intercourse, number of sexual partners, marital status or antibiotic usage.

Conculsion: The high prevalence of vaginal candidiasis among women with genital infections should be taken into account when updating policies concerning syndromic management of sexually transmitted diseases. More gender specific approach to syndromic management of sexually transmitted infections in females should be considered.
\end{abstract}

\section{INTRODUCTION}

In developing countries, much attention has been directed towards classical STD agents(1-3). This is due to the serious consequences of infections such as infertility, spontaneous abortion, still-birth, neonatal pathology and recurrent abdominal pain(4) and their possible role in enhancing the transmission of the human immune- deficiency virus(HIV)(5).

Not surprisingly, diagnosis and treatment of vaginal infections concentrate on N. gonorrhoeae, Chlamydia trachomatis, Treponema pallidum and T. vaginalis (1-4). Vaginal candidiasis (VC), although a highly prevalent condition, is a non-noticeable disease and has been excluded from the ranks of sexually transmitted diseases(6). This may be due to the mild and transitory nature of VC and the fact that it is a normal flora that is frequently isolated from healthy women with no apparent risk(7). Not surprisingly, VC has received relatively less attention by public health authorities, funding agencies and researchers(8). As a result, risk factors and pathogenic mechanisms of $\mathrm{VC}$ remain inadequately studied compared with the classical STD agents.

However, it is important to note that $\mathrm{VC}$ can cause women, their partners and to a lesser extent, their doctors considerable distress(9). VC can be associated with pruritis and offensive discharges(7), with serious social consequences resulting in profound distressing deterioration in quality of life and emotional relationships(10). In addition, it is also possible that the presence of vaginal candidiasis may alter the natural history, diagnosis or response to treatment of classical sexually transmitted infections.

In Tanzania, the risk factors associated with classical STD agents have been fairly investigated in a number of studies, but not for $\mathrm{VC}$, although it is highly prevalent $(1,11,12)$. The aim of this study was to determine the prevalence and risk factors for $\mathrm{VC}$ among women presenting at Ilala Municipal Hospital with complaints of genital infections. 


\section{MATERIALS AND METHODS}

Participants: Four hundred and sixty four women with complaints of either abnormal vaginal discharge, lower abdominal or pelvic pain or genital lesions (genital ulcer or warts), who at the time of the study (January to August 2002) were attending the outpatient clinic at Ilala Municipal Hospital in Dar es Salaam, Tanzania. Enrollment was done after obtaining an informed verbal consent. Women were excluded from the study if found to be in menstruation.

Interviews: A trained female nurse interviewed all study participants to obtain information about socio-demographic characteristics, sexual practices and other potential risk factors of VC such as use of antibiotics and past STD experiences. Gynaecological examination and sample taking: The attending physicians performed clinical examination and sample taking. Gynaecological examination looked for vulvovaginal signs and symptoms as described by Sobel et al(8). Both endocervical and high vaginal swabs were collected aseptically using sterile cotton swabs and were inserted immediately in Stuart's transport media. Patients were thereafter treated syndromically using the Ministry of Health guidelines (Tanzania).

Laboratory processing: The endocervical swabs were inoculated into modified Thayer Martin Medium (VCNT) (Difco) for isolation of $N$. gonorrhoeae, while the high vaginal swabs were inoculated on to Sabourand's dextrose agar for isolation of Candida spp. N. gonorrhoeae isolates were identified on the basis of typical colony morphology, visualization of gram-negative intracellular diplococci, positive oxidase reaction and sugar fermentation tests. C. albicans were confirmed by growth characteristics and germ tube demonstration. Both organisms were identified using standard laboratory procedures. Wet preparations of the high vaginal swabs were used for microsopic identification of $T$. vaginalis. The diagnosis of VC was established using an algorithm based on (i) symptoms (vulvar (pruritis, pain or burning sensation and dysuria) (ii) signs (vulvar erythema, edema, fissures, vaginal erythema, and thick, curdy vaginal discharge)(13) and positive wet mounts with abundance of pseudohyphae and cultures with yeast counts more than 10 colony forming units $(\mathrm{cfu} / \mathrm{ml})(14)$.

The diagnosis of bacterial vaginosis was made if $\geq 3$ of the following objective criteria were present; (i) abnormal discharge as reported by the clinicians (ii) vaginal $\mathrm{pH}>4.7$ iii) presence of clue cells, and iv) positive amine test with release of fishy odour on addition of $10 \% \mathrm{KOH}$ to vaginal secretions(15).

IgG anti HIV-1 antibodies were determined using two enzymes linked immunosorbent assays (ELISA) that are based on different principles(16). HIV testing was done using the anonymous unlinked strategy. The sera were also tested for the presence of antibodies to syphilis by both venereal diseases research laboratory (VRDL) test (Murex Biotech, Dartford, England) and Treponema pallidum haemagglutination assay(TPHA) (Fujirebio Inc. Tokyo, Japan). Syphillis was diagnosed to be recent (or active) if both VDRL (with a titre greater than 1.80) and TPHA were reactive(17). All laboratory procedures were performed in the microbiology and immunology laboratories of the Muhimbili University College of Health Sciences in Dar es Salaam, Tanzania. The average time from sample collection to laboratory processing, for the bacteriological samples, was on average 3 to 4 hours. Sera for HIV and syphilis serology were kept at $-20^{\circ} \mathrm{C}$ until the time for assay.

Statistical analysis: Data were keyed in ASCII format and imported to STATA(Stata Statistical software, op.cit.). After having corrected data entry mistake by running validation file programme in EPIINFO version 6.0 the data file was corrected for logical errors. Statistical analysis was done using SPSS statistical software version 11(19). Univariate and multiple regression were used to calculate risk estimates as odds ratio (OR) with 95\% confidence intervals (95 CI\%).

For multiple regression analysis, the best combination of independent variables associated with the outcome variable (vaginal candidiasis) was selected in a non-automated way. Variables were removed and re-entered one by one in order to assess if they added to the fit of the model with simpler models(20). The Hosmer and Lemeshow statistic was used to test the fit of the final model. A two sided $\alpha$-level $=$ 0.05 was chosen for hypothesis testing, and its complement $1-\alpha$, as the level of confidence.

Ethics: The ethics committee of the Muhimbili University College of Health Sciences gave ethical clearance. The Dar es Salaam City Medical Officer gave permission to conduct the study in the Ilala Municipal Hospital. Patients' results were handled with confidentiality and those found to be infected with classical STDs and candidiasis were treated according to the guidelines of the Ministry of Health in Tanzania.

\section{RESULTS}

The social and demographic characteristics of the study sample are presented in Table I. Most of the women were aged between 20 and 29 years, with primary education and having no employment. Participants were mainly married or cohabiting, had one sexual partner and an income of less that 50,000/Tanzanian shillings (50US Dollars), and had first sexual intercourse before 20 years of age. 
Table 1

Social and demographic characteristics of the study population $(n=464)$

\begin{tabular}{llll}
\hline Variable & & No. & $(\%)$ \\
\hline Age group & $<20$ & 52 & 11.2 \\
& $20-24$ & 151 & 32.5 \\
& $25-29$ & 109 & 23.5 \\
Educational level & $30+$ & 152 & 32.8 \\
& No formal education & 30 & 6.5 \\
Occupation & Primary & 343 & 73.9 \\
& Secondary + & 90 & 19.4 \\
No. of sex partners & Not employed & 330 & 71.1 \\
& Employed & 130 & 28.9 \\
Marital status & $\leq 1$ & 225 & 48.5 \\
& $2-3$ & 130 & 28.0 \\
Pregnancy status & $4+$ & 70 & 15.1 \\
& Single & 155 & 33.4 \\
Antibiotic use & Married/cohabiting & 287 & 61.9 \\
& Widow/separated & 21 & 4.5 \\
Income & Not pregnant & 401 & 86.4 \\
Age at first coitus & Pregnant & 61 & 13.6 \\
& Not used & 165 & 35.6 \\
& Used & 296 & 64.4 \\
& $<50000$ & 236 & 50.9 \\
& $50000+$ & 28 & 6.0 \\
& $<20$ & 335 & 72.2 \\
& $20-24$ & 85 & 18.3 \\
& $25+$ & 13 & 2.8 \\
\hline
\end{tabular}

Table 2

Risk factors for vaginal candidiasis presented as odds ratios $(O R)$ and with $95 \%$ confidence interval

\begin{tabular}{|c|c|c|c|c|c|}
\hline \multirow[t]{2}{*}{ Risk factor/Indicator } & \multirow[t]{2}{*}{ No. } & \multicolumn{2}{|c|}{$\mathrm{VC}$} & \multirow{2}{*}{$\begin{array}{l}\text { Age adjusted OR } \\
(95 \% \mathrm{CI})\end{array}$} & \multirow[t]{2}{*}{ Model OR (95\%CI) } \\
\hline & & No. & $(\%)$ & & \\
\hline \multicolumn{6}{|l|}{ Trichomonas vaginalis } \\
\hline No & 421 & 199 & 47.3 & 1.000 & 1.000 \\
\hline Yes & 43 & 11 & 25.6 & $0.383(0.19-0.78)$ & $0.263(0.12-0.57)$ \\
\hline \multicolumn{6}{|l|}{ Neisseria gonorrhoeae } \\
\hline No & 457 & 208 & 45.5 & 1.000 & 1.000 \\
\hline Yes & 7 & 2 & 28.6 & $0.479(0.09-2.49)$ & $0.279(0.05-1.55)$ \\
\hline \multicolumn{6}{|l|}{ HIV } \\
\hline No & 361 & 154 & 42.7 & 1.000 & 1.000 \\
\hline Yes & 102 & 56 & 54.9 & $1.636(1.05-2.55)$ & $1.655(1.03-2.67)$ \\
\hline \multicolumn{6}{|l|}{ Syphilis } \\
\hline No & 443 & 200 & 45.1 & 1.000 & 1.000 \\
\hline Yes & 20 & 10 & 50.0 & $1.215(0.49-2.98)$ & $1.585(0.59-4.27)$ \\
\hline \multicolumn{6}{|l|}{ Bacterial vaginosis } \\
\hline No & 238 & 87 & 36.6 & 1.000 & 1.000 \\
\hline Yes & 223 & 122 & 54.7 & $2.096(1.44-3.04)$ & $2.582(1.72-3.87)$ \\
\hline \multicolumn{6}{|l|}{ Genital discharge } \\
\hline No & 287 & 113 & 39.4 & 1.000 & \\
\hline Yes & 177 & 97 & 54.8 & $1.867(1.28-2.73)$ & $* *$ \\
\hline \multicolumn{6}{|l|}{ Genital pruritis } \\
\hline No & 192 & 71 & 37.0 & 1.000 & 1.000 \\
\hline Yes & 272 & 139 & 51.1 & $1.781(1.22-2.60)$ & $1.813(1.22-2.71)$ \\
\hline \multicolumn{6}{|l|}{ Venereal warts } \\
\hline No & 446 & 198 & 44.4 & 1.000 & \\
\hline Yes & 18 & 12 & 66.7 & $2.505(0.92-6.79)$ & - \\
\hline \multicolumn{6}{|l|}{ Genital ulcers } \\
\hline No & 394 & 175 & 44.2 & 1.000 & \\
\hline Yes & 67 & 35 & 51.5 & $1.336(0.80-2.24)$ & - \\
\hline
\end{tabular}




\begin{tabular}{|c|c|c|c|c|c|}
\hline \multicolumn{6}{|l|}{ Occupation } \\
\hline Not employed & 330 & 161 & 48.8 & 1.000 & 1.000 \\
\hline Employed & 130 & 49 & 37.7 & $0.641(0.42-0.97)$ & $0.549(0.35-0.86)$ \\
\hline \multicolumn{6}{|l|}{ Income } \\
\hline$<50000$ & 236 & 109 & 46.2 & 1.000 & \\
\hline$\geq 50000$ & 28 & 11 & 39.3 & $0.770(0.34-1.72)$ & - \\
\hline \multicolumn{6}{|l|}{ Age of first coitus } \\
\hline$<20$ & & & & 1.000 & \\
\hline $20-24$ & & & & $1.210(0.75-1.96)$ & - \\
\hline $25+$ & & & & $0.821(0.25-2.65)$ & - \\
\hline \multicolumn{6}{|l|}{ No. of sex partners } \\
\hline$\leq 1$ & 225 & 96 & 42.7 & 1.000 & \\
\hline $2-3$ & 130 & 61 & 46.9 & $1.179(0.76-1.82)$ & - \\
\hline $4+$ & 70 & 32 & 45.7 & $1.110(0.65-1.91)$ & - \\
\hline \multicolumn{6}{|l|}{ Pregnancy status } \\
\hline Not pregnant & 401 & 180 & 44.9 & 1.000 & \\
\hline Pregnant & 61 & 29 & 47.5 & $1.102(0.64-1.89)$ & \\
\hline \multicolumn{6}{|l|}{ Education status } \\
\hline No formal education & 30 & 12 & 40.0 & 1.000 & 1.000 \\
\hline Primary & 343 & 150 & 43.7 & $1.110(0.50-2.46)$ & $1.110(0.50-2.46)$ \\
\hline Secondary + & 90 & 48 & 53.3 & $0.43(0.11-0.73))$ & $0.4(0.10-0.69)$ \\
\hline \multicolumn{6}{|l|}{ Marital status } \\
\hline Single & 155 & 74 & 47.7 & 1.000 & \\
\hline Married/cohabitor & 287 & 129 & 44.9 & $0.909(0.60-1.37)$ & - \\
\hline Widow/separated & 21 & 7 & 33.3 & $0.569(0.21-1.55)$ & - \\
\hline \multicolumn{6}{|l|}{ Antibiotic use prior 2 weeks } \\
\hline No & 296 & 126 & 42.6 & 1.000 & - \\
\hline Yes & 165 & 82 & 49.7 & $1.335(0.91-1.95)$ & - \\
\hline
\end{tabular}

** Genital discharge was collinear with genital pruritis and was therefore ommitted in the final model

Of the 464 women examined, $177(38.1 \%)$ had vaginal discharge, $68(14.7 \%)$ had genital ulcers, 272 $(58.6 \%)$ had genital pruritis, $18(3.9 \%)$ had genital warts and $585(12.5 \%)$ had chancre. The prevalence of bacterial vaginosis, vaginal candidiasis, N. gonorrhoeae, HIV, syhillis and $T$. vaginalis were $48.4 \%, 45.7 \%, 1.5 \%, 22 \%$, $4.3 \%$ and $9.3 \%$ respectively. The occurrence of vaginal candidiasis were positively associated with HIV (OR= $1.6695 \% \mathrm{CI}(1.1-2.7)$, bacterial vaginosis $(\mathrm{OR}=2.6$, $95 \% \mathrm{CI}(1.7-3.8)$, genital prurities $(\mathrm{OR}=1.8,95 \% \mathrm{CI}$ $(1.2-2.7)$ and negatively associated with $\mathrm{TV}$ OR $=0.3$, $95 \%$ CI $(0.1$ - 0.6), occupation $(\mathrm{OR}=0.549,95 \%$ $\mathrm{CI}=(0.35-0.86)$ and with secondary education $(\mathrm{OR}=$ $0.43,95 \% \mathrm{CI}=(0.11-0.73)$. There was increased but non-significant odds for $\mathrm{VC}$ with venereal warts and syphilis. There was no association between $\mathrm{VC}$ and N. gonorrhoeae, genital ulcers, age at first intercourse, number of sexual partners, educational status, marital status or antibiotic usage.

\section{DISCUSSION}

This study aimed to determine the prevalence and risk factors for vaginal candidiasis among women with genital infections who were seeking care at a municipal hospital in Dar es Salaam, the capital city of Tanzania. It is important, for targeted intervention, to note that most of the patients were young (20 and 29 years), with only primary education and having no employment and had first sexual intercourse before 20 years of age. This combination of factors makes these women fall in the high risk group for HIV infection and other STDs.
Our findings indicate that nearly half $(45.7 \%)$ of the women seeking primary care for genital infections had vaginal candidiasis, being the second most prevalent condition after bacterial vaginosis $(48.4 \%)$. This findings needs to be taken into account when screening patients for genital infections and in updating national guidelines for the treatment of sexually transmitted infections. To this end, the Candida spp responsible for vaginal candidiasis needs to be determined together with their in-vitro susceptibility to antifungal agents.

The high prevalence of bacterial vaginosis in our study underscores the importance for routine screening of patients with simple tests such as determination of vaginal $\mathrm{pH}$ and microscopy for clue cells. In one study(22), empiric treatment of genital infection with metronidazole based on $\mathrm{pH}$ measurement alone was found to be clinically effective in $89 \%$ of patients. Such a strategy is feasible in Tanzania and could be applied in antenatal clinics, family planning centres, in STD as well as outpatient clinics attending patients with genital infections.

It is worth noting that over one fifth of patients were HIV seropositive, which compares well with other studies done in various populations and hospital settings (Table 3). This finding gives further evidence for the high prevalence in the sexually active population in Tanzania and does suggest the need to promote HIV testing and counselling services in settings seeing patients with genital infections.

The low prevalence of active syphilis (4.3\%) and gonococcal infections (1.5\%) found in this study is in keeping with findings of studies in Dar es Salaam 
among pregnant women attending antenatal clinics(1) and those attending family planning clinics(11) and could be due to the fact that these infections are normally the main focus in screening and treatment of women attending antenatal clinics, where majority $(>90 \%)$ of women of reproductive age normally attend. The prevalence of $T$. vaginalis was higher $(9.3 \%)$ than that of $N$. gonorrhoeae and syphilis, which compares with other studies in the country $(11,12)$. This finding could be related with prescription tendencies frequently involving the use of antibiotics alone without metronidazole(23), which may also explain the high prevalence of bacterial vaginosis seen in this study.

Regarding the association between occurrence of VC and risk factors, it was associated with HIV - 1 infection, bacterial vaginosis and genital pruritis, which is in keeping with the reports in literature (8). The significant association between $\mathrm{VC}$ and education level supports the high impact of female education and sexual and reproductive health(24). Given the early age of sexual debut ( $<20$ years) seen in this study, this education need to be started very early, possibly in adolescence, before the girls are sexually active.

The association between VC and occupation seen in this study seem to imply that effective STD intervention programmes need to add an element of economic empowerment to be more effective. Interestingly, there was no association between $\mathrm{VC}$ and factors such as $N$. gonorrhoeae, genital ulcers, age at first intercourse, and number of sexual partners, marital status or antibiotic usage. The lack of association between VC and age at first sexual intercourse, number of sexual partners, marital status has been reported in some other studies $(11,25)$, making it difficult to predict occurrence of VC based on sexual and social characteristics of women.

The lack of association between vaginal candidiasis and either syphilis or $N$. gonorrhoeae could be explained by the rather low prevalence of the two infections in the studied population. Antibiotic usage, a factor known to be associated with $\mathrm{VC}$, was not a significant factor in this study, probably due to the fact that most women with history of antibiotic usage normally take a single course or are even not able to afford the whole dose (23).

The fact that VC was associated with various clinical presentations (genital discharge, pruritis) and that there was no clear association between $\mathrm{VC}$ and sexual or other putative factors complicates innovation of a screening algorithm for VC and calls for use of laboratory services whenever possible.

In conclusion, the high prevalence of $\mathrm{VC}$ and bacterial vaginosis among females with genital infections calls for a gender specific guidelines to syndromic detention and management of sexually transmitted infections. There is a need for training health laboratory workers in antenatal, STD, outpatient and family planning clinics in simple screening techniques such as microscopy and $\mathrm{pH}$ determination in order to improve the detection of $\mathrm{VC}$ and $\mathrm{BV}$ cases, which may often be asymptomatic and therefore missed.

Given the high prevalence of vaginal candidiasis among patients with genital infections(45.7\%) we recommend further studies to elucidate the type(s) of Candida spp involved, their in-vitro antifungal susceptibility pattern, as well as to determine clinical response following antifungal treatment.

\section{ACKNOWLEDGEMENTS}

We acknowledge, with gratitude, the support of doctors and nurses of the Outpatient Department of the Ilala Municipal Hospital and laboratory assistance accorded to us by the staff of the Department of Microbiology and Immunology of the Muhimbili University College of Health Sciences (MUCHS). We wish to thank all patients who participated.

\section{REFERENCES}

1. Mwakagile, D., Swai, A.B.M., Sandstrom, E., Biberfeld, G. and Mhalu, F.S. High frequency of sexually transmitted diseases among pregnant women in Dar es Salaam, Tanzania: Need for intervention. East Afr. Med. J. 1996; 73: 675-658.

2. Cu-Uvin, S., Hogan, J.W., Warren, D., et al. Prevalence of lower genital tract infections among human immunodeficiency virus (HIV) - seropositive and high risk HIV- seronegative women. Clin. infect. Dis. 1999; 29:11451150 .

3. Fonck, K., Kidula, N., Jaoko, W. et al. Validity of vaginal discharge algorithm among pregnant and non-pregnant women in Nairobi, Kenya. Sex. Transm. Infect. 2000; 76:33-38.

4. Passey, M., Mgone, C.S., Lupiwa, S. et al. Community based study of sexually transmitted diseases in rural women in the highlands of Papua New Guinea: prevalence and risk factors. Sex Transm. Infect. 1998; 74:120-127.

5. Orroth, K.K., Gavyole. A., Todd, J., Mosha, F. et al. Syndromic treatment of sexually transmitted diseases reduces the proportion of incident HIV infections attributable to these diseases in rural Tanzania. AIDS 2000; 14:1429-1437.

6. Sobel, J.D. Vulvovaginitis. When Candida becomes a problem. Dermatol Clin. 1998; 16:763-768.

7. Sobel, J.D. Pathogenesis and treatment of recurrent vulvovaginal candidiasis. Clin. Inf. Dis. 1992; 14:148-153.

8. Sobel, J.D., Faro, S., Force, R.W. et al. Vulvovaginal candidiasis: epidemiologic, diagnostic and therapeutic considerations. Amer. J. Obstet .Gynecol 1998; 78:203-211.

9. O'Neill, S. and Howard, J. Recurrent vulvovaginal candidiasis. A gynaecological enigma. Aust. Fam. Physician 1989; 18:99-102.

10. Sobel, J.D. Candidal vulvovaginitis. Clin. Obstet. Gynecol 1993; 36:153-165.

11. Gerting, D.M., Kapiga, S.H., Shao, J.F. and Hunter, D.J. Risk factors for sexually transmitted diseases among women attending family planning clinics in Dar es Salaam, Tanzania. Genitourin Med. 1997; 73:39-43.

12. Mayaud, P., Ka -Gina, G. and Cornelissen, J. et al. Validation of a WHO algorithm with risk assessment for the clinical management of vaginal discharge in Mwanza, Tanzania. Sex Transm. Infect. 1998; 74:77-84 
13. Eckert, L.O., Hawes, S.E., Stevens, C.E. et al. Vulvovaginal candidiasis: clinical manifestations, risk factors, management algorithm. Obstet Gynecol 1998; 92:757-765.

14. Hopwood, V., Crowley. T., Horrock, C.T. et al. Vaginal candidiasis: relationship between counts and symptoms and clinical signs in non-pregnant women. Genitourin Med. 1988; 64:331-334.

15. Amsel, R., Totten, P.A., Spiegel, C.A. et al. Non especific vaginitis: diagnostic criteria and microbial and epidemiological association. Amer. J. Med. 1983; 74:14-22.

16. Urassa, W., Godoy, K., Killewo, et al. The accuracy of alternative confirmatory strategy for detection of antibodies to HIV-1: experience from a regional laboratory in Kagera, Tanzania. J. Clin. Virol. 1999; 14:25-29.

17. Urassa, W., Kapiga, S.H., Msamanga, G.I. et al. Risk factors for syphilis among HIV-1 infected pregnant women in Dar es Salaam, Tanzania. Afr. Reprod. Health. 2001; 5:54-62.

18. Dean, A. G., Dean J. A., Coulombier D. et al. Epi Info Version 6.0: A word processing Database and Statistical system. Atlanta, GA: Centres for Disease Control 1994.
19. The Statistical Package for the Social Sciences (2001). Release 11.0 SPSS Inc: Chicago.

20. Hosmer, D.W. and Lemshow, S. Applied logistic regression. New York: John Wiley and Sons, 1989.

21. Grosskurth, H., Mwijarubi, E., Todd, J. et al. Operational performance of an STD control programme in Mwanza region, Tanzania. Sex Transm. Infect. 2000; 76:426-136

22. Riordan, T., Macaulay, M.E., James, J.M. et al. A prospective study of genital infections in a family-planning clinic 1. Microbiological findings and their association with vaginal symptoms. Epidemiol. Infect. 1990; 104:47-53.

23. Matee, M.I.N. Appropriateness of antimicrobial prescriptions at Muhimbili National Hospital in Dar es Salaam, Tanzania. Tanz Med. J. (in press).

24. Blankahart, D., Muller, O., Gresenguet G. and Weis, P. Sexually transmitted infections in young pregnant women in Bangui, Central Africa Republic. Int. J. STD AIDS 1999; 10:609-614.

25. Reed, B.D., Gorenflo, D.W., Gillespie, B.W., Pierson, C.L. and Zazove, P. Sexual behaviours and other risk factors for Candida vulvovaginitis. J. Women Health Gend Based Med. 2000; 6:645-655. 